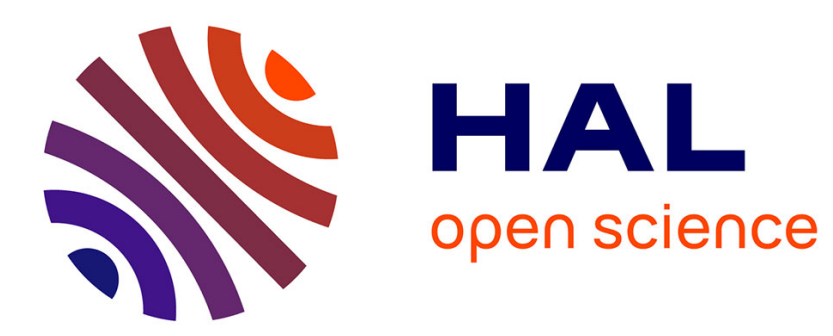

\title{
Influence of dislocation cores on the plasticity of materials
}

\author{
F. Louchet
}

\section{To cite this version:}

F. Louchet. Influence of dislocation cores on the plasticity of materials. Journal de Physique IV Proceedings, 1993, 03 (C7), pp.C7-567-C7-574. 10.1051/jp4:1993794 • jpa-00252214

\section{HAL Id: jpa-00252214 https://hal.science/jpa-00252214}

Submitted on 1 Jan 1993

HAL is a multi-disciplinary open access archive for the deposit and dissemination of scientific research documents, whether they are published or not. The documents may come from teaching and research institutions in France or abroad, or from public or private research centers.
L'archive ouverte pluridisciplinaire HAL, est destinée au dépôt et à la diffusion de documents scientifiques de niveau recherche, publiés ou non, émanant des établissements d'enseignement et de recherche français ou étrangers, des laboratoires publics ou privés. 


\title{
Influence of dislocation cores on the plasticity of materials
}

\author{
F. LOUCHET
}

LTPCM-ENSEEG, Institut National Polytechnique de Grenoble, BP. 75, 38402 St Martin d'Hères cedex, France

\section{I- INTRODUCTION:}

For the sake of brevity, this paper will not be a comprehensive review, but will rather focus on some recent results on the influence of core structure on kinetics of dislocation glide, and in particular in the cases where lattice friction arises either from cross-slip or non-planar dissociation of dislocations, or from strong covalent (or ionocovalent) bonding. These quite different types of "friction" find expression in various types of dislocation locking, which will be discussed and compared, including the influence of temperature.

\section{II- CROSS-SLIP AND NON-PLANAR DISSOCIATIONS:}

In many structures (BCC, close packed hexagonal (CPH), various intermetallics...) dislocations are dissociated in a non planar way, or experience frequent cross-slip between two types of slip planes. These dissociated dislocations are most often screws (a/2<111> in BCC, a/3 $<11-20>$ in CPH, a $<110>$ in L12,...), but can occasionally be edges (e.g. Lomer-Cottrell locks in FCC). Their core structures and their relations with plasticity have been either extensively studied $(1,2)$, or still under discussion, as in a large variety of intermetallics $(3,4,5)$.These dissociations strongly hinder dislocation motion, at least at low temperatures, since local constrictions are necessary to nucleate double kinks (dk) before kinks can move apart.

The observation of dislocation motion in thin foils by in situ TEM experiments show that the movement can be either smooth or jerky. Dislocations usually move in a smooth and viscous way in BCC metals, in prismatic planes of CPH structures (6), and in cube planes of several L12 ordered alloys. In contrast, a jerky motion can be observed in a number of different situations: in BCC metals, (Nb, FeCr: (7)), jumps of screws are often correlated. In other cases, non correlated jumps are observed, probably resulting from an intrinsic property of dislocations: this is the case for prismatic glide in $\mathrm{Be}$ at room temperature and in $\mathrm{Mg}$ at low temperatures (8), in prismatic and 1st order pyramidal planes of Ti,. Such a behaviour has also been reported for glide in cube planes of a CMSX2 superalloy between 400 and $1400^{\circ} \mathrm{C}(9)$, but not in a Ni3A1 crystal of a similar.composition as the ordered phase. Finally, a kind of jerky motion of nearly screw but not really straight dislocations is also observed (e.g. in FeCr), and will be discussed in connection with the stress anomaly of some intermetallics.

\section{II-1- SMOOTH MOTION:}

The typical example is that of BCC metals. All experimental data are consistent with a dk mechanism which brings screw dislocations into the next valley in which they dissociate again. There is no evidence in this particular case for any friction on kinks, and the resulting dislocation velocity is observed to vary linearly with the dislocation length (e.g. Film "Living Metals", by T. Imura). This is exactly what is expected from a $\mathrm{dk}$ mechanism in which the mean free path of kinks is larger than the average dislocation length: a single $\mathrm{dk}$ brings the dislocation into the next valley, and the velocity is expected to be proportional to the number of dk nucleation sites, i.e. to the dislocation length:

$$
v=v_{D} b^{2} \mathbf{L} / c^{2} \exp (-\Delta G(\tau) / k T)
$$

where $v_{D}$ is the Debye frequency, $b$ the Bürgers vector modulus, $L$ the length of the screw dislocation, $1 c$ the size of the critical $\mathrm{dk}, \Delta \mathrm{G}$ the activation energy for $\mathrm{dk}$ 
nucleation. However, as mentioned above, some jerky motion has been occasionally observed. This is the case of $\mathrm{Fe} \mathrm{Cr}$ where the jerky motion cannot be ascribed to a metastable glissile state of the dislocation core: dislocations paths between two stops are wavy, which means that screws change their slip planes continuously by successive dissociations and $\mathrm{dk}$ nucleations in each atomic row (7). The reason for this jerky movement can probably be understood as a collective motion of interacting straight and parallel dislocations.

\section{II-2 - JERKY MOTION:}

We mean here intrinsic jerky motion, i.e. uncorrelated jumps of dislocations directly related to their core properties. The dislocation can be found in two different "states" with different mobilities.

A first (anecdotic?) type is that of softening of Fe by $\mathrm{C}$ interstitials $(10)$, where $\mathrm{C}$ atoms were supposed to help dk nucleation: at low temperatures, screw dislocations are long enough to find $\mathrm{C}$ atoms in most Peierls valleys ; they move rapidly by easy dk nucleation until they find a valley without any $C$ atom, in which they stop for a while. This situation is characterized by two different waiting times depending on the type of valley found on the dislocation path.

A second type assumes the existence of a metastable glissile state for the dislocation core, and is known as the "locking-unlocking" (L-UL) mechanism (11). The difference with the previous one is that here all the sites are equivalent, and that the dislocation can be in either state in every atomic row. If $\Delta \mathrm{Gul}$ and $\Delta \mathrm{Gl}$ are respectively the activation energies for unlocking the dislocation from the "sessile" state and to lock it again from the glissile to the sessile state, the corresponding probabilities are:

$$
\begin{aligned}
& \mathrm{Pul}=v_{\mathrm{D}}\left(\mathrm{bL} / \mathrm{lc}^{2}\right) \exp (-\Delta \mathrm{Gul} / \mathrm{kT}) \\
& \mathrm{Pl}=v_{\mathrm{D}}\left(\mathrm{bL} / \mathrm{l}^{2} \mathrm{c}^{2}\right) \exp (-\Delta \mathrm{Gl} / \mathrm{kT})
\end{aligned}
$$

where $\mathrm{lc}$ and $\mathrm{l}^{\prime} \mathrm{c}$ are the sizes of the corresponding critical double kinks.

The unlocking and the locking probabilities have been supposed to be proportional to the dislocation length (11). Though this is quite reasonable for the straight dislocation in the locked configuration, this is not obvious in the glissile state of the fast expanding loop, but the qualitative conclusions would not be drastically affected.

Experimentally, the lifetime of the glissile configuration is found much smaller than the rest time in the sessile state, which means that Pul $\ll$ Pl. The resulting velocity is then:

$$
\mathrm{v}=\operatorname{vo}(\tau) \mathrm{Pul} / \mathrm{Pl}=\operatorname{vo}(\tau)(\mathrm{l} \mathrm{c} / \mathrm{lc})^{2} \exp (-(\Delta \mathrm{Gul}-\Delta \mathrm{Gl}) / \mathrm{kT})
$$

where $\tau$ is the resolved shear stress, and vo $(\tau)$ is the instantaneous velocity of the dislocation in the glissile state.

The main consequence of this model comes from the observation that Pul $\ll$ Pl. According to (11), this suggests $\Delta \mathrm{Gul}>\Delta \mathrm{Gl}$, and despite the fact that jerky motion is often associated with stress anomaly, no intrinsic stress anomaly can be expected in this case. Additional ingredients are then needed: a variation of the stacking fault energy with temperature has first been invoked, but an interesting and more recent model involves a variation with temperature of the cross-slip amplitude of the trailing partial (12) . Nevertheless, one can wonder whether the small value of Pul compared to Pl cannot be compensated by a large value of the ratio of the prefactors $(\mathrm{lc} / \mathrm{l} \mathrm{l}) 2$, which would allow DGul.-DGl $<0$ in spite of Pul $<$ Pl, and yield consequently an anomalous temperature dependence of the stress.

\section{II-3- FROM THE INDIVIDUAL TO THE COLLECTIVE BEHAVIOUR:}

The L-UL mechanism (and this is perhaps suggested by some in situ observations) deals with an isolated single straight segment of dislocation which is assumed to be alternatively and as a whole either in the stable state or in the metastable one. In contrast, in a bulk crystal, stable and metastable configurations are likely to be found 
along a given dislocation at a given time. This is equivalent to local pinning and unpinning. A recent simulation for L12 alloys (13) assumes that pinning occurs randomly by cross-slip from (111) to (010) planes, with a probability which increases with the screw character of the superdislocation. The resulting configurations consist in highly pinned segments close to the screw direction, connected by superkinks (sk), and are similar to those commonly observed in deformed specimens. Superdislocations proceed by lateral motion of superkinks, as also observed for a slightly different reason in heavily jogged dislocations in a $\mathrm{BCC}$ alloy (FeCr (14)) : jogs resulting from crossslip of the screw dislocation in different planes play the same pinning role as the crossslipped segments in the present simulation. Due to this type of motion, and in contrast with the L-UL mechanism, the average velocity of a near-screw segment is always a significant fraction of the free flight velocity. Arising from fluctuations in sk density, some dislocations can become immobile. This exhaustion mechanism which obviously increases with temperature leads in a very natural way to the stress anomaly. The main difference with the previous type of models is that here the strain rate is much more controlled by the instantaneous density of mobile dislocations rather than by their velocities. This very convincing approach has the advantage to take into account in a statistical way the whole dislocation population through its collective response to the external stress, and is probably applicable to a number of other cases.

\section{II-4- EFFECT OF TEMPERATURE:}

II-4-1- From jerky to smooth motion:

In the frame of the L-UL mechanism, the jump length is related to the lifetime $\delta \mathrm{tj}$ of the glissile state by:

$$
\lambda \mathrm{j}=\mathrm{vo}(\tau) \delta \mathrm{tj}=\mathrm{vo}(\tau)(1 / \mathrm{Pl})
$$

which decreases (at constant stress) when temperature increases, and eventually reaches the interatomic spacing. In this limiting case, the dislocation locks in every valley. We are brought back to the Peierls mechanism, which appears here as a high temperature limiting case of the locking-unlocking mechanism.

\section{II-4-2- From individual to interactive motion:}

In both cases of Peierls and of "L-UL" mechanisms, dislocations are usually stiff and behave in an individual way as long as the stress necessary to move them is larger than their mutual interactions. At higher temperatures, and above the stress anomaly for materials prone to it, the flow stress $\sigma(T)$ is a decreasing function of temperature at constant strain rate. A "stiff-soft" transition is expected when $\sigma(\mathrm{T})=\alpha \mu \mathrm{b} \rho 1 / 2$, above which dislocations become smooth-shaped (15), and move jerkily in a collective way, as they do in most FCC metals.

\section{III- BREAKING STRONG BONDS:}

A second main category of materials in which core structures have a strong influence on dislocation mobilities is those in which bonding is of covalent or ionocovalent nature, like elemental semiconductors (ESC) (Si, Ge, Diamond), compound semiconductors (CSC) (III-V and II-VI compounds), covalent ceramics, ....Breaking such bonds is necessary to nucleate double kinks or even to move kinks. We shall discuss here the different possible core structures and their consequences on mobility, taking as an example the case of semiconductors.

\section{III-1- DIFFERENT POSSIBLE CORE STRUCTURES:}

ESC have a diamond cubic structure, consisting of two interpenetrating FCC lattices, displaced from one another by one quarter of the cube diagonal. Dislocations loops 
have usually an hexagonal shape, exhibiting two screw segments parallel to the Bürgers vector $\mathrm{a} / 2\langle 110\rangle$, and four $60^{\circ}$ segments aligned along the two other $\langle 110\rangle$ directions of the (111) slip plane. In the case of CSC, two different structures are found. In the sphalerite (cubic) structure, the two atoms of the basis are of different chemical nature. The wurtzite (hexagonal) structure differs from the previous one by the stacking sequence of compact planes (ABAB instead of $A B C A B C$ ). Though we shall discuss here the cubic case, the results can be transposed easily to the hexagonal case. A more thourough study of this problem can be found in (16) .

The core structure of the perfect dislocation was first discussed by Hornstra (17). For the same Bürgers vector, two different cores can exist, depending on whether the extra half plane ends between widely spaced (shuffle set) or narrowly spaced (glide set) pairs of (111) planes. A glide core (G) can tranform into a shuffle one (S) by addition of a row of vacancies (Sv core) or of interstitials (Si core) (18), leading to the following types of reactions:

$$
\begin{aligned}
& \mathrm{G}+\mathrm{i} \rightarrow \mathrm{Si} \\
& \mathrm{G}+\mathrm{v}>\mathrm{Sv} \\
& \mathrm{Si}+2 \mathrm{v} \rightarrow \mathrm{Sv}
\end{aligned}
$$

Due to the periodicity of the lattice along the vertical $<111>$ axis, Si and Sv cores are identical. They contain a single dangling bond $(\mathrm{db})$ per period instead of 3 for the $G$ core, and is then expected to be more stable, though a number of $G$ sites in thermodynamical equilibrium cannot be excluded (19).

However, dislocations are always dissociated, with a stacking fault lying in the $G$ plane, since a dissociation in the $S$ plane would usually lead to a high energy stacking fault (20).

The simplest configuration is that of a dissociated $\mathrm{G}$ dislocation, shown in fig $1: 30^{\circ} \mathrm{G}$ shockley partials have a single db per period, but $90^{\circ} \mathrm{G}$ partials have a "double comb" structure with $2 \mathrm{db}$ per period. The plane view of fig 1 shows that, in CSC, core atoms are of different nature according to the type of partial. Screw segments are made of two $30^{\circ}$ partials of different natures, whereas $90^{\circ}$ partials consist of one $30^{\circ}$ and one $90^{\circ}$ partial of similar chemical natures, leading to the so-called $\alpha$ and $\beta$ dislocations (for details on this nomenclature, see the proceedings of the Hünfeld conference (21). It is worth noticing from fig 1 that the structure of a kink on a given partial is identical to the structure of the neighbouring partial parallel to the kink. Breaking bonds is necessary to move kinks, and a large migration energy $\mathrm{Wm}$ of kinks is then expected.

Dangling bonds in both types of shockley $G$ partials can be reconstructed, at least in ESC, as shown schematically in fig 2. Such a reconstruction doubles the spatial period of the $30^{\circ}$ core, changing a broad half-filled band covering the whole gap into a narrow filled band close to the edge of the valence band, and a narrow empty band close to the conduction band, thus decreasing significantly the electronic energy. Electronic energy calculations confirm the stability of the $30^{\circ}$ core, but also of the $90^{\circ}$ one (22). This tendency to reconstruction is expected to increase the migration energy of kinks on this type of partial, since the reconstruction energy has to be added to the "normal" migration energy of the kink. Reconstruction defects (antiphase sites), called solitons, can be imagined along reconstructed cores (23). Reconstruction is probably more difficult in CSC, due to the same chemical nature of core atoms in the core.

As for perfect dislocations, rows of vacancies or interstitials can be added directly to the cores of $30^{\circ}$ and $90^{\circ}$ Shockley partials (16). But now, since we start from the dissociated state as a reference, Si and Sv partials are no more identical. Different types of $\mathrm{Si}$ and $\mathrm{Sv}$ cores are discussed in (16).

Though the first high resolution images in Si gave reasonable evidence for a $\mathbf{G}$ structure, at least for the $30^{\circ}$ partial (24), more detailed investigations showed that the situation is not so simple (25). Although $30^{\circ}$ partials appear very straight at the scale of weak-beam observations their contrasts in HREM are blurred and not reproducible, and could result from a number of isolated or clustered Sv and Si sites along the "average" $\mathrm{G}$ core. In contrast, $90^{\circ}$ cores are remarkably reproducible, and fit Marklund's simulations of a G $90^{\circ}$ core (26), but a few shuffle sites (more likely Sv for steric reasons) cannot be excluded. 


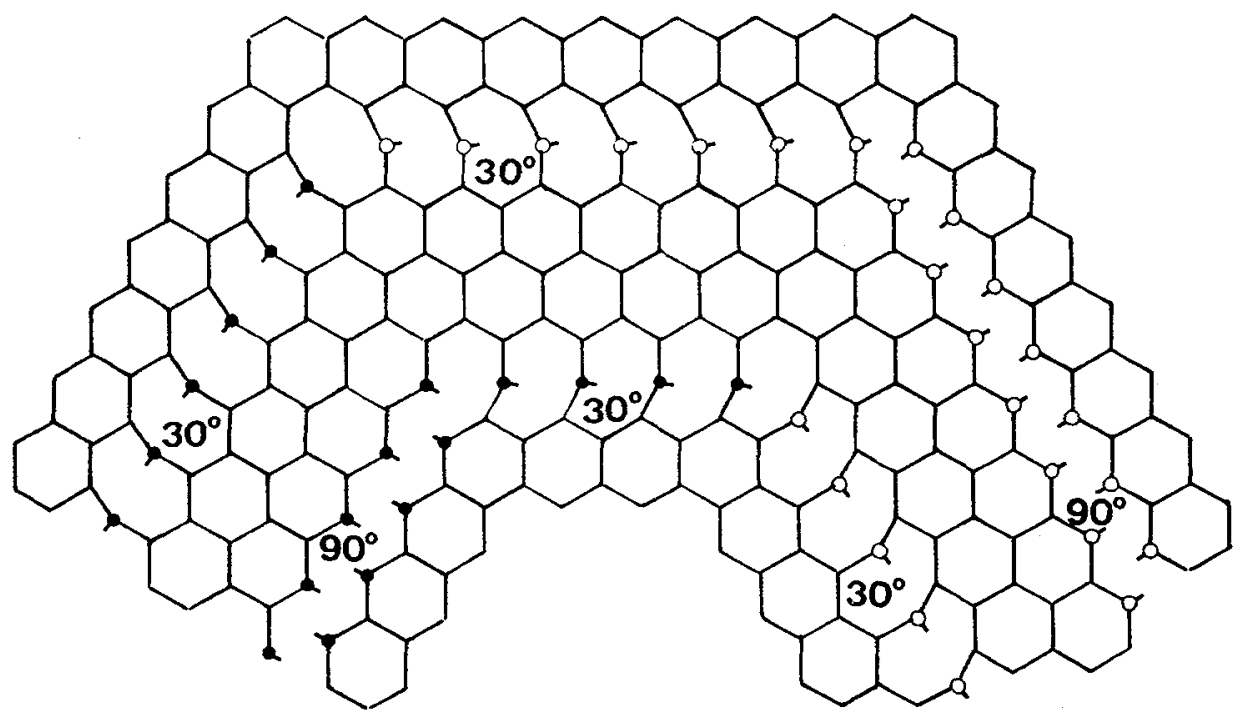

Fig 1: Plane view of a dissociated half-loop in the glide set. White and black atoms respectly refer to the two interpenetrating FCC lattices.

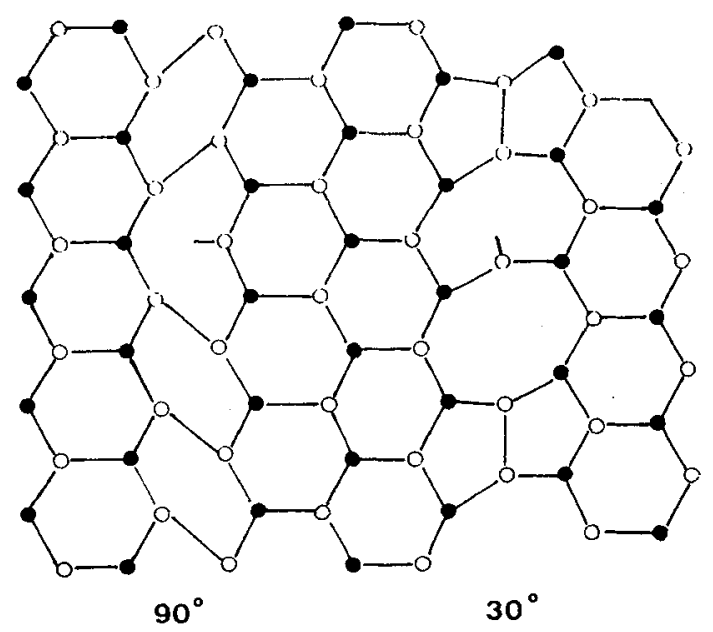

Fig 2: Typical reconstructions of a $90^{\circ}$ and of a $30^{\circ}$ partial, showing a soliton on each of them. 


\section{III-2- DISLOCATION MOBILITY:}

Dislocation mobility in semiconductors, mainly in Silicon, has been investigated extensively by different techniques. Most results can be found in the proceedings of the Hünfeld conference $(21)$, and in $(27,28,29)$. We shall focuss here on more recent results, mainly obtained on $\mathrm{Ge}$ and in compound semiconductors, and in most cases by in situ straining in a transmission microscope.

In this type of experiments, dislocation loops are shown to expand smoothly (jerky motion is never observed), keeping their hexagonal shapes, at least at moderate temperatures, which gives reasonable evidence for a double-kink (dk) mechanism.

Measurements of dislocation velocities as a function of dislocation lengths $L$, were first performed in Si (30) (31), and later in Ge (32). They showed a linear increase for short dislocations, but a saturation at large lengths, in agreement with the two velocity regimes predicted by Hirth and Lothe in the case of a lattice friction Wm on kinks (18). The values of the kink formation energy $\mathrm{Fk}$ and of the kink migration energy Wm have been roughly estimated in Si by a measurement of the mean free path of kinks (about $0.4 \mu \mathrm{m}$ at $\mathrm{T}=540^{\circ} \mathrm{C}, \sigma=550 \mathrm{MPa}$ ), and more accurately in Ge through a fit in the intermediate length regime. In Si, Fk is found around $0.4 \mathrm{eV}$, and $\mathrm{Wm}$ between 1.2 and $1.3 \mathrm{eV}$. In Ge, Fk is $0.5 \mathrm{eV}$ for $60^{\circ}$ dislocations and 0.55 for screws, Wm is around 0.9 $\mathrm{eV}$ for $60^{\circ}$ dislocations and between 0.75 and $0.8 \mathrm{eV}$ for screws. These measurements stand for dissociated dislocations and not for partials, but the figures found for screws are likely to be sound for $30^{\circ}$ partials, since they are made of two such partials. This suggests that kink migration might be slightly easier on $30^{\circ}$ than on $90^{\circ}$ partials, in contradiction with the easier reconstruction of this type of partial. The complex "blurred" $30^{\circ}$ core invoked above might be responsible for such a behaviour : Sv sites, i.e. broken bonds, could help kink migration along the $30^{\circ}$ core. However, these problems are far from being understood, and further investigations are needed.

In CSC, velocity measurements show a tremendous difference between very fast $\alpha$ dislocations (V (i.e. B) type atoms in a III-V CSC) and much slower $\beta$ (A atoms) and screws $(29)$. The above analysis could suggest (i) a less reconstructed core (i.e. a low $\mathrm{Wm}$ ) of the fast $30^{\circ}$ and $90^{\circ} \alpha$ partials into which the $60 \alpha$ dislocation is dissociated, but, for unknown reasons, this should not be the case (at least to the same extent) of the $\beta$ partials. A second possibility should be (ii), still for obscure reasons, a particularly low mobility of the $30^{\circ} \beta$, whose core is made of $A$ atoms, and which is the common partial belonging to the slowest (screw and $60^{\circ} \beta$ ) dislocations. A third possibility should be (iii) an understoechiometry of the a cores (Sv sites) due to the larger vapour pressure of the (V or VI) B elements, which could help kink nucleation or migration.

In these compounds, a linear dependence on $\mathrm{L}$ of velocities of the slowest dislocations is observed in the whole investigated length range (up to $3 \mu \mathrm{m}$ in GaAs), which suggests a lower migration energy Wm as compared to $\mathrm{ESC}(29)$, and probably due to a more difficult reconstruction of the core as stated above.

In ESC and CSC, dislocations are often quite sensitive to electron or photon radiation (cathodoplastic and photoplastic effects). Since the experiments performed by Kisielowski-Kemmerich (33) in Si for the photoplastic effect, and by Maeda (34) in GaAs for the cathodoplastic effect, a number of new results have been obtained, in particular for the cathodoplastic effect in II-VI compounds. In the case of $\mathrm{ZnS}$ for instance (29), dislocation, velocities are multiplied by a factor 2500 for an electron beam density of $1000 \mathrm{~A} / \mathrm{m}^{2}$. This increase of mobility shows a tendency to saturation for large current densities. The cathodoplastic effect in these materials is generally ascribed to the production of electron-hole pairs under electron irradiation : these pairs can recombine preferentially at dislocations in a non-radiative way, thus helping $\mathrm{dk}$ nucleation (35). 


\section{III-3- EFFECT OF TEMPERATURE:}

As in the case of non-planar dissociated cores, a stiff-soft transition can be expected for a temperature Tc such that $\sigma(\mathrm{T})=\alpha \mu \mathrm{b} \rho^{1 / 2}$. This transition has been actually observed, in particular in $\mathrm{Ge}$ : above $\mathrm{Tc}$, dislocations are softer and move in a collective way (7). However, some kinetic aspects of dislocation motion are still strongly influenced by lattice friction, even very close to the melting point. A typical example is the case of non attractive forest cutting in Ge. The activation energy $\Delta \mathrm{G}$ is usually related to the average waiting time $\Delta t$ by:

$$
\Delta \mathrm{G}=\mathrm{kT} \ln \left(\mathrm{v}_{\mathrm{D}} \mathrm{b} / 1 \Delta \mathrm{t}\right)
$$

where $l$ is the obstacle separation. Taking an activation volume $\mathrm{v}^{*}=1 b^{2}$, the maximum activation energy $\Delta$ Go can be deduced from direct measurements of $\Delta t$. This leads to tremendous values of $\Delta \mathrm{Go}$ (about $50 \mathrm{eV}$ !) at temperatures between 550 and $700^{\circ} \mathrm{C}$ (i.e. between 0.7 and $0.8 \mathrm{Tm}$ ) (7). The reason why this classical model does not apply here can be understood by comparing the vibration frequency $\left(v_{D} b / l\right)$ of a dislocation of length 1 to the jump frequency $\left(V_{D} \exp (-W m / k T)\right)$ of a kink submitted to a lattice friction. This gives a critical length $1^{*}$ above which dislocation vibrations are slow enough not to be hindered by kink drift, and below which they are too fast to operate freely. In the present case, $1^{*}$ has been estimated between 6 to $30 \mu \mathrm{m}$, obviously much larger than the observed dislocation lengths. It can be concluded that lattice friction on kinks hinder thermally activated cutting of non-attractive trees up to temperatures very close to the melting point (the value of $T$ for which $1^{*}$ would equal the measured value of the forest spacing 1 is slightly larger than the melting point!)

\section{IV-CONCLUSION:}

We have illustrated the large variety of dislocation cores which can generate lattice friction and their consequences on plasticity by some generic examples. In covalent and ionocovalent crystals, though some fundamental questions are still open, plasticity at low temperatures proceeds by smooth and individual motion of dislocations and can be considered as reasonably understood. At high temperatures however, Peierls forces vanish in such a way that kink mobility is still hindered even close to the melting point, and can modify significantly high temperature plasticity.

In the case where lattice resistance arises from cross-slip or non planar dissociation of dislocations, smooth or jerky motion of dislocations can be observed, but local pinning and depinning along nearly screw dislocations (although perhaps more difficult to evidence in thin foils) could be a rather general feature of dislocation motion. Macroscopic plasticity of these materials, and more specifically of the large variety of intermetallics, is still far from being understood. No doubt that core structure studies are still essential, but investigations of dislocation behaviour at different scales, taking into account collective effects, seem to be a compulsory passage from the mobility of a single (and sometimes short) dislocation to the (sometimes strange) plasticity of the macroscopic crystal.

\section{REFERENCES:}

(1) Vitek V., Crystal Lattice Defects 5 (1974), 1.

(2) Duesbery M.S., in "Dislocations in Solids", ed. F.R.N. Nabarro, North Holland, Amsterdam, vol. 8 (1989), 67.

(3) Veyssière P., Revue de Physique Appliquée 23 (1988), 431.

(4)Baluc N.L., PhD thesis, Ecole Polytechnique Fédérale de Lausanne (Switzerland), (1990).

(5) Hemker K., PhD thesis, Stanford University (USA)(1990). 
(6) Couret A., Caillard D., Acta Metall. 36 (1988), 2515.

(7) F. Louchet, A Triki and J. Pelissier, Proc. of the France-Japan Seminar on in situ Electron Microscopy, in "Microscopy, Microanalysis, Microstructures", under press (1993).

(8) Couret A., Caillard D., Puschl W., Schoeck G., Phil. Mag. 63 (1991), 1045.

(9) N. Clément, A. Couret, D. Caillard, Phil. Mag. (1991).

(10) Kubin L.P., Louchet F., Acta Metall. 27 (1979), 337.

(11) D. Caillard, A. Couret, N. Clément, S. Farenc, G. Molenat, Proc.ICSMA 9, Haifa (1991), 139.

(12) Molénat G., Caillard D., colloque plasticité 93, Villeneuve d'Ascq.

(13) Mills M.J., Chrzan D.C., Acta Metall. Mater., 40 (1992), 3051.

(14) Triki A., Louchet F., unpublished results.

(15) Louchet F., Kubin L.P., Phil. Mag. A, 39 (1979), 433.

(16) Louchet F., Thibault-Desseaux J., Revue de Physique Appliqué 22 (1987), 207.

(17) Hornstra J. ,J. Phys. Chem. Solids 5, (1958) 129.

(18) Hirth J.P. and Lothe J. , Theory of Dislocations, Mc. Graw Hill (1968).

(19) Blanc J., Phil. Mag. 32, (1975) 1023.

(20) Meingast R., Alexander H. , Phys. Stat. Sol.(a), 17, 229 (1973).

(21) Int. Symposium on Dislocations in Tetrahedrally Coordinated Semiconductors, J. Phys. Colloq. 40 (1979), C-6.

(22) Marklund S. J. Phys. Colloq. 44 (1983), C4-25.

(23) Heggie M., Jones R., J. Phys. Colloq. 44 (1983), C4-43.

(24) Anstis G., Hirsch P.B., Humphreys C., Hutchison J., Ourmazd A., Inst. Phys. Conf. Series 60 (1981), 15.

(25) Bourret A., Desseaux-Thibault J., Lançon F., J. Phys. Colloq., 44 (1983), C4-15.

(26) Marklund S., Phys. Status Solidi (b), 100 (1980), 77.

(27) Louchet F., George A., J. Phys. Colloq. 44 (1983), C4-51.

(28) Alexander H., in "Dislocations in Solids", F.R.N. Nabarro ed., vol..8 .

(29) Louchet F., Pélissier J., Caillard D., Peyrade J.P., Levade C., Vanderschaeve G., Proc. of the France-Japan Seminar on in situ Electron Microscopy, in "Microscopy, Microanalysis, Microstructures", under press (1993).

(30) Louchet F., Inst Phys. Conf. Series 60 (1981), 35.

(31) Hirsch P.B., Ourmazd A., Pirouz P., Inst Phys. Conf. Series 60 (1981), 29.

(32) Louchet F., Cochet-Muchy D., Bréchet Y., Pélissier J., Phil. Mag. A, 57, n² (1988), 327.

(33) Alexander H., Kisielowski-Kemmerich C. and Weber E.R., ICDS 12, Amsterdam 1982, Physica B.

(34) Maeda K., Takeuchi S., J. Phys. Colloq. 44 (1983), C4-375)

(35) Ourmazd A., Weber E., Gottschalk H., Booker G.R., Alexander H., Inst Phys. Conf. Series 60 (1981), 63. 